Article

\title{
Analysis of the Distribution of Some Potentially Harmful Elements (PHEs) in the Krugersdorp Game Reserve, Gauteng, South Africa
}

\author{
Michael Shapi ${ }^{1}$, Maryam Amra Jordaan ${ }^{1, *}$, Devandren Subramoney Nadasan ${ }^{1}$, \\ Theophilus C. Davies ${ }^{2}$ (), Emmanuel Chirenje ${ }^{3}$, Mpumelelo Dube ${ }^{3}$ and Mammusa R. Lekoa ${ }^{4}$ \\ 1 Faculty of Natural Sciences, Mangosuthu University of Technology, 511 Mangosuthu Highway, Umlazi, \\ Durban 4031, KwaZulu-Natal Province, South Africa; mshapi@mut.ac.za (M.S.); \\ Nadasan@mut.ac.za (D.S.N.) \\ 2 Department of Geology, University of Nigeria, Nsukka, Nsukka 410001, Nigeria; theo.clavellpr3@gmail.com \\ 3 Council for Geoscience, Tswane 0184, South Africa; echirenje@geoscience.org.za (E.C.); \\ mdube@geoscience.org.za (M.D.) \\ 4 Research and Innovation Department, Community Engagement Unit, University of Zululand, \\ KwaDlangezwa, Vulindlela Road, Richards Bay 3886, South Africa; LekoaM@unizulu.ac.za \\ * Correspondence: maryamamrajordaan@gmail.com; Tel.: +27-31-907-7269; Fax: +27-86-616-3453
}

Received: 1 December 2019; Accepted: 19 December 2019; Published: 10 February 2020

\begin{abstract}
The Mintails Mogale Gold (MMG) and the Rand Uranium (RU) are two large-scale mining consortiums active in re-mining old tailings dams and dumps in Krugersdorp and are a source of mine discharge feed into the Krugersdorp Game Reserve (KGR). This has resulted in a noticeable accumulation of potentially harmful elements (PHEs) over a number of years. Efforts were implemented to interpret the concentration levels of PHEs in soils of the study areas of which a total of 36 georeferenced soil samples were collected (in triplicate) from the MMG, RU and KGR, including samples from farmlands and waterways adjacent to the mining sites. Samples were then analysed by both inductively coupled plasma optical emission spectrometry (ICP-OES) and inductively coupled plasma mass spectrometry (ICP-MS) for 36 elements. From the 36 elements of this study, detailed evaluations of the occurrence of 12 selected elements were discussed. The geochemical landscape at the KGR is shown to be in flux. The major mediating influences on the behaviour of $\mathrm{As}, \mathrm{Co}, \mathrm{Cu}$, $\mathrm{Hg}$ and $\mathrm{Pb}$, as they enter the KGR largely in the form of acid mine drainage (AMD), are the geological substrate (mostly in carbonate form). Analysis of the soils showed high levels of contamination for As and Co in ppm. The mean maximum of As ranged from (5.00-170.30) with the highest level found in the Krugersdorp site. The mean maximum of Co ranged from (46.00-102.30) with the highest level found in MMG. All of these values were well above the recommended maximum acceptable concentration (MAC) values, i.e., As (15-20) and Co (20-50). The mean maximum values for $\mathrm{Pb}$ (12.40-92.30); Cu (18.50-115.30) and $\mathrm{Hg}$ (12.40-92.30) content in surface soils of all four segments studied falls well within the MAC range for agricultural soils i.e., $\mathrm{Cu}(60-150) ; \mathrm{Hg}(0.5-5)$ and $\mathrm{Pb}$ (20-300).
\end{abstract}

Keywords: potentially harmful elements (PHEs); Krugersdorp; maximum acceptable concentration (MAC); acid mine drainage (AMD)

\section{Introduction}

Krugersdorp Game Reserve (KGR) in the Gauteng Province of South Africa is one of the country's most important wildlife parks in terms of the revenue it generates from tourism and related activities. The Reserve is juxtaposed to two major mining outfits, Mintails Mogale Gold (MMG) and Rand 
Uranium (RU) at Krugersdorp, and smallholdings (SH). This makes the total environment an ideal setting for investigating the impact of mining and ore processing activities on the health of neighbouring wildlife communities, and other nearby ecosystem components.

Krugersdorp $\left(26^{\circ} 6^{\prime} 0^{\prime \prime} S ; 27^{\circ} 46^{\prime} 0^{\prime \prime} \mathrm{E}\right)$, Figure 1, also referred to as Mogale City, is located in the West Rand of the Gauteng Province of South Africa. It has a population of close to half a million inhabitants. It is flecked with a number of abandoned gold (with $\mathrm{Mn}, \mathrm{Fe}$, asbestos and lime) mines and boasts an industry that contributes significantly to the economic activity of the Gauteng region. MMG and RU are two large-scale mining consortiums active in re-mining of old tailings dams and dumps in Krugersdorp.

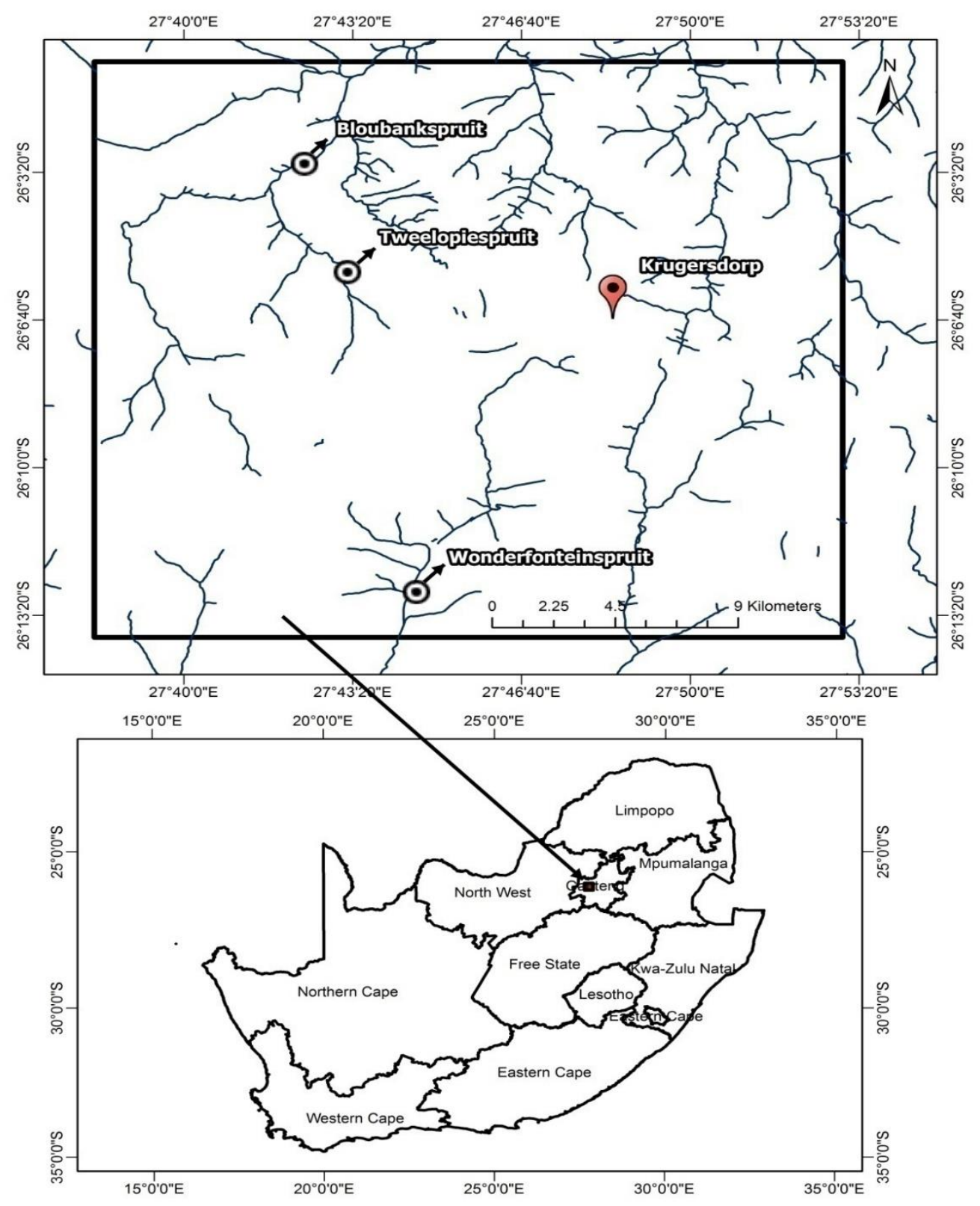

Figure 1. The study area.

Although a comprehensive literature search revealed extensive published information on the hydrochemical environment and soil geochemistry at MMG, RU and $\mathrm{SH}$, as well as wildlife health at KGR, information regarding any on soil geochemistry is scarce [1]. The main aim of this study was to 
provide a comprehensive data base of the occurrence and levels of potentially harmful elements (PHEs) in the study area, which is not available anywhere, rather than only focus on a few selected elements.

Heavy metals are a natural occurrence in nature all around the world or as a result of anthropogenic activities through emissions from the rapidly escalating industrial areas, mine tailings, discarding of high metal wastes and atmospheric deposition [1-4]. The metals commonly found in soil as a result of these activities include copper $(\mathrm{Cu})$, lead $(\mathrm{Pb})$, zinc $(\mathrm{Zn})$, nickel $(\mathrm{Ni})$, cobalt $(\mathrm{Co})$, mercury $(\mathrm{Hg})$ and cadmium (Cd). Some of these metals are essential in small doses for use in living organisms for their normal physiological functioning. However, large concentrations can tend to destabilize ecosystems due to bioaccumulation in organisms causing toxic effects through metabolic interference and mutagenesis [1,5-7]. In addition, their accumulation in soils persists for a long period of time after their introduction, contributing to contamination of ecosystems $[1,8,9]$. Arsenic in particular, is quite toxic and readily absorbed by plants from groundwater and soil [10]. Mining of gold minerals, as seen in the mining consortiums in Krugersdorp is related to acid drainage problems that can cause long-term damage to waterways and biodiversity. Acid mine drainage (AMD) occurs when sulfide-bearing material are exposed to oxygen and water and typically ensues in iron sulfide-aggregated rocks. Although this process occurs naturally, mining can stimulate AMD generation merely through increasing the measure of sulfides exposed. Furthermore, naturally-occurring bacteria can hasten AMD production by supporting the breakdown of sulfide minerals [11]. The obvious concern is the deterioration of water and soil quality from the impact of AMD from the West Rand Basin, which inspired the need to garner more detailed information on the distribution characteristics and fate of the heavy metals around the mining area at Krugersdorp and to establish their levels of concentration. The above considerations provided motivation for the present study, which is aimed at investigating and documenting the PHE- and other element additions from mine effluents (AMD). This study reports on the distribution and concentration of heavy metals due to RU and MMG mining activities in the Krugersdorp region and their migration to surrounding smallholdings and the KGR, focusing on cobalt $(\mathrm{Co})$, mercury $(\mathrm{Hg})$, aluminium $(\mathrm{Al})$, zinc $(\mathrm{Zn})$, copper $(\mathrm{Cu})$, nickel $(\mathrm{Ni})$, lead $(\mathrm{Pb})$, cadmium $(\mathrm{Cd})$ and arsenic (As). This information is vital in formulating intervention measures such as mitigation (AMD neutralisation), rehabilitation and soil amendment.

Soils

Soils of Krugersdorp are mainly reddish brown to red and belong to the Hutton form (orthic topsoil on red apedal subsoil, usually on rock). The soils range in texture from clayey silt through silty clay to loam. During the field studies, sandy clay was also encountered at a few sites, but the sand content was always less than $10 \%$. The soils are predominantly light textured ( $15 \%-25 \%$ clay), and vary in depth from less than $400 \mathrm{~mm}$ to over $1.2 \mathrm{~m}$.

The KGR is primarily grassland, with shrub thickets, small forests and fynbos found in the valley and close to the small dams. The only information that refers to soil quality, and is relevant to the present study, is the description of agricultural potential of the proposed route of the Westgate substation, west of KGR [12].

Although Krugersdorp is designated as an agricultural hub in the Gauteng Province, the general potential for agricultural development remains relatively low. Based on soil factors (not climatic conditions), Paterson (2008) describes areas of moderate or high agricultural soils [13].

\section{Materials and Methods}

\subsection{Sampling Technique and Sample Preparation}

A comprehensive literature search was used to garner published information on the hydrochemical environment and soil geochemistry at MMG, RU and SH. Some data were found to exist on hydrochemistry and wildlife health [1], but hardly any on soil geochemistry. Therefore, in order to complement the existing hydrochemical data, a total of 36 soil samples were collected for analysis (in 
triplicate) from cultivable and uncultivable lands at the MMG and RU gold mine fields, and the KGR and adjacent $\mathrm{SH}$ to provide information on the soil geochemistry.

The map of the study area with sample sites is shown in Figure 2. At the mining sites (MMG and RU) soil samples were collected on a grid using pre-determined Global Positioning System (GPS) points. Adjacent to the mines and at the KGR, soil samples were taken at random intervals and at a distance of $15 \mathrm{~m}$ on either side of minor roads or footpaths (not shown) running approximately in the direction of rivers draining the two mining areas into the KGR (Figure 2). A distance of $15 \mathrm{~m}$ on either side of the paths was considered sufficient in minimizing anthropogenic influence on element fluxes in soils in the non-cultivable sectors of the land.

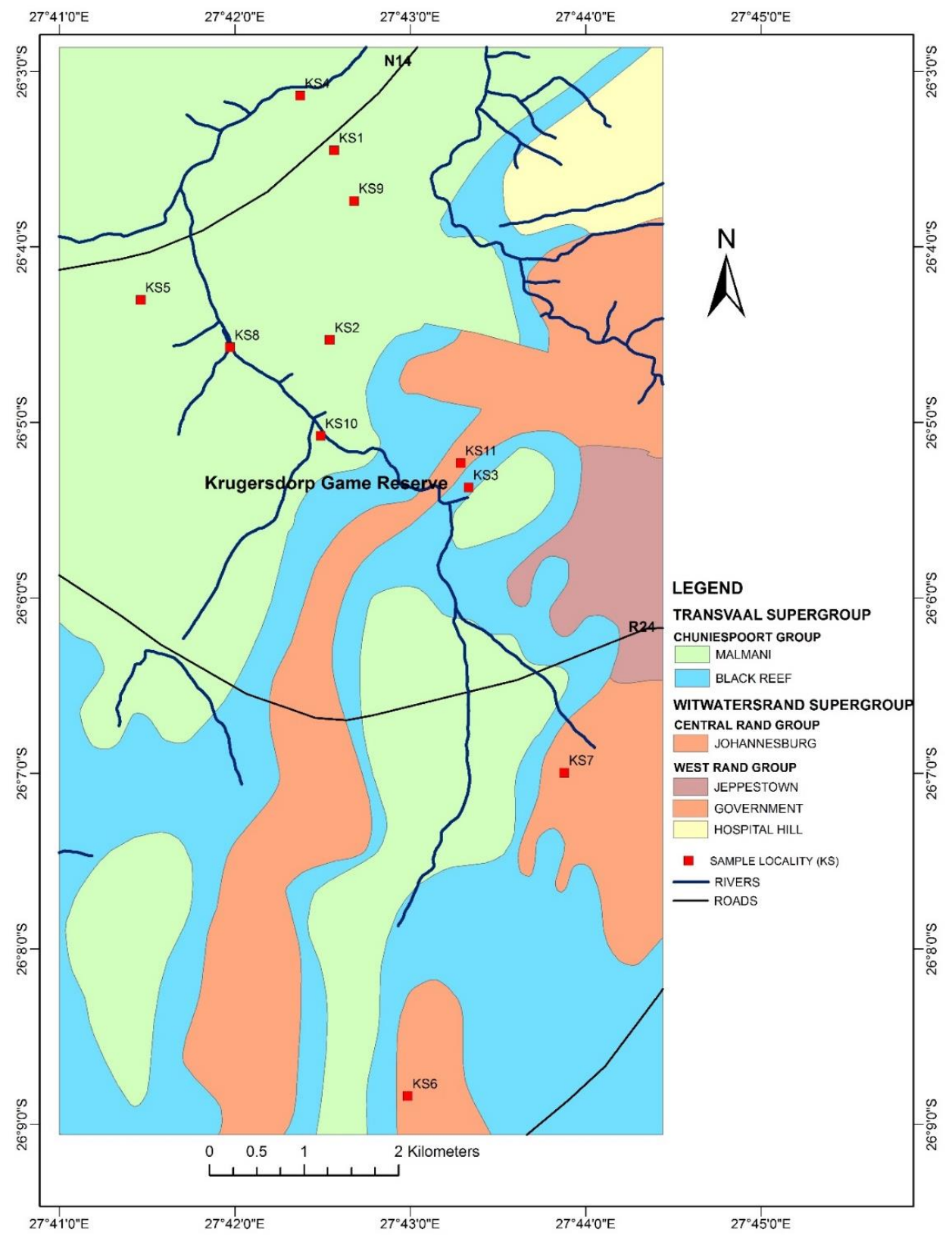

Figure 2. Geology and soil sampling points. $\mathrm{KS}=$ soil sampling points.

This sample design was selected in order to determine concentration levels and migration characteristics of PHEs in pollution trails emanating from the mine sites and entering the Krugersdorp ecosystem, to provide a comprehensive data base of the occurrence and levels of PHEs in the study area. 
The GPS coordinates as well as other geoenvironmental characteristics such as geology, topography, vegetation, pedology and hydrological characteristics were carefully recorded at each sampling site. The soil sampling protocol comprised of the construction in an undisturbed area, of a $1 \mathrm{~m} \times 1 \mathrm{~m}$ pit to a depth of $40 \mathrm{~cm}$ using pick and shovel. A composite near-surface soil sample ( $30 \mathrm{~cm}$ below A0) was collected and stored in pre-labelled Kraft paper envelopes in accordance with The International Geological Correlation Programme (IGCP) 259 recommendations [13], modified for application in tropical conditions by [14].

\subsection{Soil Sample Preparation and Analysis}

Each soil sample was oven-dried $\left(30^{\circ} \mathrm{C}\right)$ for about $12 \mathrm{~h}$, homogenized and then quartered in the Biochemistry Laboratories of the Mangosuthu University of Technology. Following disaggregation, the soil samples were sieved to $<180 \mu \mathrm{m}$ ( 80 mesh). The samples were then stored in plastic vials and shipped to the headquarters of ACME Laboratories in Vancouver, Canada, for analyses. ACME's sample preparation method comprised of the weighing out of a $30 \mathrm{~g}$ split followed by an Aqua Regia dissolution technique. Following soil sample digestion, a range of nutritional and PHEs were determined by inductively coupled plasma optical emission spectrometry (ICP-OES) and inductively coupled plasma mass spectrometry (ICP-MS) techniques, but only the PHEs of $\mathrm{As}, \mathrm{Co}, \mathrm{Cu}, \mathrm{Hg}$ and $\mathrm{Pb}$ were selected for this study.

\subsection{Quality Assurance Evaluation}

Commercially available standards and previously analysed internal standards, as well as reagent blanks were included to check accuracy and precision. Triplicates were randomly incorporated into all analytical batches. The data were accepted if the relative standard deviation was $<15 \%$ at five times the limit of detection for the triplicate samples. Triplicate samples comprised $16 \%$ of total samples. Standard solutions of all the elements were prepared by diluting their corresponding $1000 \mathrm{mg} / \mathrm{L}$ certified standard solutions. The blank reagents and standard reference materials (SRMs) of the metals were used to verify the accuracy and precision of digestion, while in view of data quality assurance, each sample batch was analysed in triplicate under standard opt-ionizing conditions within the confidence limit of $95 \%$ (see www.acmelab.com for further details).

\subsection{Soil Fraction Analysed}

The fine fraction of $180 \mu \mathrm{m}$ ( 80 mesh) was considered suitable for the present study. A number of studies have determined that metals in the coarse-grained soils are mainly derived from parent materials, and metals in the fine fraction are mostly anthropogenic $[15,16]$. Other studies $[5,17-19]$ have classified the fraction where enrichment of PHEs take place in (contaminated) soils as clay- and silt-sized. The high specific surface area and charge of fine particles are the probable factors that account for the high contamination levels seen in the fine fraction [20]. The Aqua Regia technique used for decomposition of soil samples (in this study) is a partial dissolution procedure able to release metals associated with a recent pollution source, and could be released to the soil solution with input of water (non-silicate-bound forms); but would not dissolve metals associated within the solid phases, i.e., within the structure of the insoluble minerals [21]. As such, the method is deemed suitable for the assessment or prediction of crop plant metal content, plant growth and plant deficiency or toxicity $[22,23]$. The method could therefore be considered a suitable tool in the assessment of the long-term potential risk of heavy or toxic metals entering the biosphere.

\section{Results and Discussion}

As pointed out in the introduction, the main aim of this study was to provide a comprehensive data base of the occurrence and levels of PHEs in the study area, which is not available anywhere, rather than only focus on a few selected elements (refer to Table 1). These are presented in Tables 2-4. 


\subsection{Total Content of Selected Heavy Metals}

Some soil pollution water sources and parameters are sighted in this section. February 2011 evidence of ADM in the Krugersdorp area involving the Tweelopie stream is shown in Figure 3. Previous (2006) KGR water hydrochemical parameters are outlined in Table 1 [24]. The general analytical results of the thirty-six (36) elements in Table 2 range from $9.22 \%$ (for Fe) to less than $0.1 \mathrm{ppm}$. From the 36 elements of this study, detailed evaluations of the occurrence of 12 selected elements are presented in Table 3. Of these, five heavy metals, namely $\mathrm{As}, \mathrm{Co}, \mathrm{Cu}, \mathrm{Hg}$ and $\mathrm{Pb}$, the data of which is presented in Table 4, from the respective study sites, were considered for a comparative analysis of their concentrations in the soil samples. The concentrations obtained in the study sites are compared with the reference values for background concentrations, Johnson [25] after Wedepohl [26].

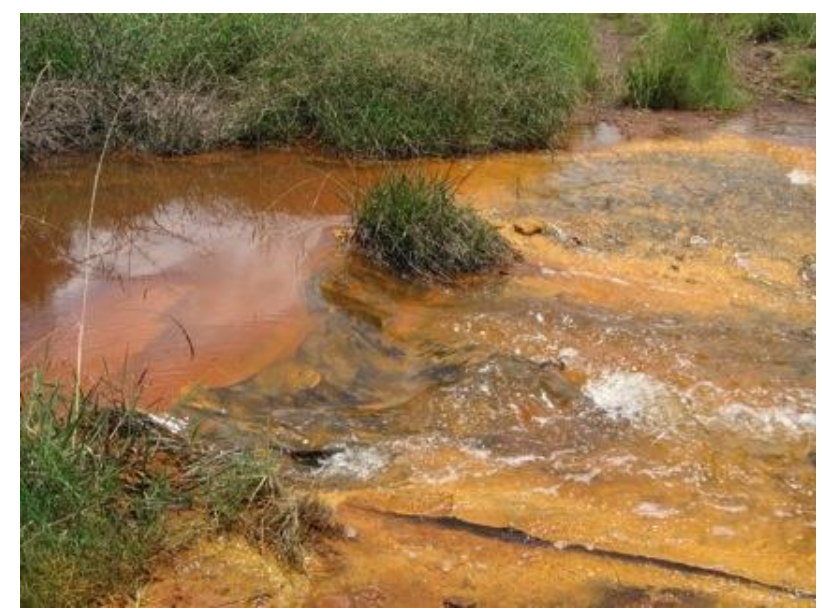

Figure 3. Tweelopiespruit (TLS) flowing out of Aviary Dam, Krugersdorp Game Reserve (KGR, 3 Feb 2011). Source: [27].

Table 1. Hydrochemical parameters of water from the KGR inlet (i.e., "treated water") for August and September 2006 in relation to the Department of water Affairs and Forestry (DWAF) Directive requirements. Source: Du Toit, 2006 [24].

\begin{tabular}{cccc}
\hline Variables & Directive & August, 2006 & September, 2006 \\
\hline $\mathrm{pH}$ & $6.5-8.4$ & $5.1^{*}$ & $9.2^{*}$ \\
Conductivity $(\mathrm{EC})(\mathrm{ms} / \mathrm{m})$ & 70 & $493^{*}$ & $438^{*}$ \\
Sulphate $(\mathrm{SO} 4)(\mathrm{mg} / \mathrm{L})$ & 200 & $3230^{* * * * *}$ & $3000^{* * * * *}$ \\
Chloride $(\mathrm{Cl})(\mathrm{mg} / \mathrm{L})$ & 36 & $50^{*}$ & $43^{*}$ \\
Aluminium $(\mathrm{Al})(\mathrm{mg} / \mathrm{L})$ & 0.06 & $0.70^{*}$ & $0.88^{*}$ \\
Managnese $(\mathrm{Mn})(\mathrm{mg} / \mathrm{L})$ & 0.07 & $85.18^{* * * * *}$ & $18.28^{* * * * *}$ \\
Iron $(\mathrm{Fe})(\mathrm{mg} / \mathrm{L})$ & 0.1 & $74.67^{* * * * *}$ & $12.47^{* * * * *}$ \\
\hline
\end{tabular}

Note: *-Not complying with DWAF's Directive; **-Not complying with the Minimum Requirements of DWAF for Livestock Watering; ${ }^{* * *}$-Not complying with the Minimum Requirements of DWAF for Domestic Use.

In the KGR, surrounding smallholdings and RU, Mn had the highest mean concentration followed by $\mathrm{Cr}$. These three study sites also had a similar trend for the four lowest heavy metal concentrations, $\mathrm{Pb}$ $>\mathrm{As}>\mathrm{Cd}>\mathrm{Hg}$ (Tables 3 and 4). The soil samples from the KGR had mean heavy metal concentrations of 93.41, 4280.33, 17.37, 64.73, 35.07, 88.56, 0.17, 0.12, 11.55, and 7.56 mg. $\mathrm{kg}^{-1}$ for Cr, Mn, Co, Ni, Cu, Zn, $\mathrm{Cd}, \mathrm{Hg}, \mathrm{Pb}$, and As respectively. In the soils from $\mathrm{MMG}$, the trend was $\mathrm{Mn}>\mathrm{Zn}>\mathrm{As}>\mathrm{Cr}>\mathrm{Cu}>\mathrm{Ni}>$ $\mathrm{Pb}>\mathrm{Co}>\mathrm{Cd}>\mathrm{Hg}$ (Table 3). The higher standard deviation reveals higher variations in heavy metal distributions from the point source of discharge to the adjacent areas. 
Table 2. Full table of Krugersdorp Game Reserve soil samples analytical potentially harmful elements (PHEs) results.

\begin{tabular}{|c|c|c|c|c|c|c|c|c|c|c|c|c|c|c|}
\hline & & Analyte & Mo & $\mathrm{Cu}$ & $\mathrm{Pb}$ & $\mathrm{Zn}$ & $\mathrm{Ag}$ & $\mathrm{Ni}$ & Co & Mn & $\mathrm{Fe}$ & As & $\mathrm{Au}$ & Th \\
\hline & & Unit & PPM & PPM & PPM & PPM & PPM & PPM & PPM & PPM & $\%$ & PPM & PPB & PPM \\
\hline & & MDL & 0.1 & 0.1 & 0.1 & 1 & 0.1 & 0.1 & 0.1 & 1 & 0.01 & 0.5 & 0.5 & 0.1 \\
\hline & Sample & Type & & & & & & & & & & & & \\
\hline 1 & KS1A & Soil & 0.9 & 15.8 & 5 & 23 & $<0.1$ & 29.5 & 11 & 2679 & 3.69 & 6.8 & 2.6 & 2.7 \\
\hline 2 & KS1B & Soil & 1 & 11.8 & 4.8 & 15 & $<0.1$ & 24 & 11.2 & 3128 & 2.74 & 5.2 & 3.6 & 2.2 \\
\hline 3 & KS1C & Soil & 1 & 16 & 6.4 & 18 & $<0.1$ & 26.3 & 11.6 & 2648 & 3.4 & 6.1 & 2.9 & 3.6 \\
\hline 4 & KS1D & Soil & 0.9 & 10.1 & 4.6 & 15 & $<0.1$ & 16.4 & 9.3 & 1801 & 2.14 & 5.2 & 3.8 & 2.2 \\
\hline 5 & KS2A & Soil & 0.5 & 16 & 7.5 & 25 & $<0.1$ & 38.5 & 10.5 & 5056 & 2.38 & 4.1 & 4.9 & 3 \\
\hline 6 & KS2B & Soil & 1.1 & 29.5 & 15.1 & 54 & $<0.1$ & 91.1 & 18 & $>10,000$ & 4.24 & 5.5 & 7.9 & 4 \\
\hline 7 & KS2C & Soil & 0.8 & 27.9 & 15.8 & 79 & $<0.1$ & 97.1 & 14.4 & $>10,000$ & 4.92 & 6.6 & 12.4 & 6.1 \\
\hline 8 & $\mathrm{KS} 2 \mathrm{C} 2$ & Soil & 0.8 & 28.7 & 16.4 & 84 & $<0.1$ & 97.2 & 14.9 & $>10,000$ & 4.74 & 6.7 & 11.8 & 5.7 \\
\hline 9 & KS2D & Soil & 1.6 & 42 & 13.5 & 216 & $<0.1$ & 444.8 & 15.3 & $>10,000$ & 8.23 & 8.6 & 8.8 & 4.4 \\
\hline 10 & KS3A1 & Soil & 0.9 & 40.6 & 6.3 & 25 & $<0.1$ & 49.3 & 11.1 & 3625 & 4.15 & 6.5 & 15.7 & 4 \\
\hline 11 & KS3A2 & Soil & 0.7 & 41.1 & 6.5 & 27 & $<0.1$ & 50.3 & 11.8 & 3960 & 4.23 & 6.4 & 15.5 & 4.2 \\
\hline 13 & KS3B & Soil & 0.9 & 43.1 & 7.1 & 30 & $<0.1$ & 61.4 & 11 & 9233 & 4.32 & 7.4 & 13.1 & 4.1 \\
\hline 14 & KS3C & Soil & 0.8 & 43.4 & 10.2 & 54 & $<0.1$ & 54.5 & 10.8 & 2956 & 4.85 & 7.3 & 14.6 & 5 \\
\hline 15 & KS3D & Soil & 0.9 & 49 & 10.6 & 54 & $<0.1$ & 59.9 & 14.1 & 3401 & 4.85 & 7.6 & 11.1 & 4.5 \\
\hline 16 & KS4A & Soil & 1 & 29.8 & 12.4 & 202 & $<0.1$ & 273.1 & 93 & 7644 & 2.86 & 6.4 & 442.2 & 2.8 \\
\hline 17 & KS4B & Soil & 0.9 & 13.5 & 7 & 12 & $<0.1$ & 23.2 & 10.4 & 3226 & 2.59 & 3.6 & 4.7 & 3 \\
\hline 18 & KS5A & Soil & 0.7 & 20.1 & 9.2 & 13 & $<0.1$ & 21.7 & 11.4 & 1558 & 2.95 & 4 & 4 & 4.8 \\
\hline 19 & KS5B & Soil & 0.5 & 16.1 & 5.9 & 12 & $<0.1$ & 19.6 & 6.4 & 1082 & 2.47 & 3.3 & 3.8 & 4.3 \\
\hline 20 & KS6A & Soil & 0.7 & 18.5 & 12.4 & 15 & $<0.1$ & 29.7 & 16.2 & 812 & 3.23 & 5 & 5.5 & 4.9 \\
\hline 21 & KS6B & Soil & 0.9 & 13.7 & 6.3 & 32 & $<0.1$ & 61.5 & 46 & 451 & 2.1 & 2.9 & 3.4 & 3.6 \\
\hline 22 & KS7A & Soil & 1.9 & 122.9 & 72.7 & 153 & 0.2 & 86.8 & 48.4 & 7086 & 3.74 & 140.7 & 272.8 & 4 \\
\hline 23 & KS7B1 & Soil & 1.7 & 115.3 & 92.3 & 1129 & 0.2 & 97.6 & 48.3 & 3011 & 4.31 & 170.3 & 407.7 & 7.1 \\
\hline 24 & KS7B2 & Soil & 1 & 61 & 50 & 690 & 0.1 & 59.6 & 26.9 & 1717 & 3.55 & 79.2 & 329.4 & 6.7 \\
\hline 25 & KS8A & Soil & 0.7 & 80 & 18.4 & 736 & $<0.1$ & 110.2 & 48.7 & 9290 & 4.01 & 16.8 & 354.9 & 3.2 \\
\hline 26 & KS8B & Soil & 0.8 & 91.4 & 14.6 & 281 & $<0.1$ & 77.4 & 102.3 & 7866 & 3.87 & 14.1 & 257.2 & 3.2 \\
\hline 27 & KS9A & Soil & 0.9 & 18.6 & 7 & 19 & $<0.1$ & 29.5 & 13.7 & 2366 & 2.71 & 4.7 & 15.4 & 3.7 \\
\hline 28 & KS9B & Soil & 0.9 & 17.6 & 8.2 & 21 & $<0.1$ & 27.5 & 13.3 & 2628 & 2.44 & 4.7 & 16.3 & 3.6 \\
\hline 29 & KS9C & Soil & 0.6 & 20 & 5.2 & 16 & $<0.1$ & 30.4 & 10.3 & 1807 & 2.82 & 3.9 & 21.1 & 4.3 \\
\hline 30 & KS9D & Soil & 0.7 & 24 & 3.6 & 17 & $<0.1$ & 31.6 & 9 & 1122 & 3.4 & 3.2 & 13.2 & 4.7 \\
\hline 31 & KS $10 \mathrm{~A}$ & Soil & 1 & 93.2 & 79.6 & 446 & 4.7 & 117.6 & 39.4 & 8990 & 4.27 & 15.9 & 488.7 & 2.2 \\
\hline 32 & KS 11 Anthill sample & Soil & 1 & 47.3 & 13.2 & 33 & $<0.1$ & 39.3 & 11.8 & 527 & 6.39 & 7.5 & 60 & 2.9 \\
\hline 33 & KS $11 \mathrm{~A} 1$ & Soil & 0.8 & 18.8 & 6.3 & 16 & $<0.1$ & 19.8 & 4.8 & 256 & 2.83 & 11.5 & 26.5 & 2 \\
\hline 34 & KS 11 A2 & Soil & 1 & 18.5 & 6.1 & 15 & $<0.1$ & 18.6 & 4.5 & 212 & 2.62 & 11.6 & 30.7 & 1.9 \\
\hline 35 & KS 11 B & Soil & 0.7 & 11.1 & 3.6 & 10 & $<0.1$ & 13.9 & 2.8 & 115 & 1.67 & 7.6 & 19.6 & 1.5 \\
\hline 36 & KS $11 \mathrm{C}$ & Soil & 0.8 & 36 & 6.6 & 26 & $<0.1$ & 39.6 & 12.9 & 630 & 9.22 & 4.2 & 18.7 & 3 \\
\hline 37 & KS $11 \mathrm{D}$ & Soil & 1.1 & 55.3 & 9.6 & 36 & $<0.1$ & 51.9 & 20.6 & 1273 & 9.08 & 8.5 & 30 & 3.8 \\
\hline
\end{tabular}


Table 2. Cont

\begin{tabular}{|c|c|c|c|c|c|c|c|c|c|c|c|c|c|c|}
\hline & & Analyte & Mo & $\mathrm{Cu}$ & $\mathrm{Pb}$ & $\mathrm{Zn}$ & Ag & $\mathrm{Ni}$ & Co & Mn & $\mathrm{Fe}$ & As & $\mathrm{Au}$ & Th \\
\hline & & Unit & PPM & PPM & PPM & PPM & PPM & PPM & PPM & PPM & $\%$ & PPM & PPB & PPM \\
\hline & & MDL & 0.1 & 0.1 & 0.1 & 1 & 0.1 & 0.1 & 0.1 & 1 & 0.01 & 0.5 & 0.5 & 0.1 \\
\hline & Sample & Type & & & & & & & & & & & & \\
\hline 38 & * Pulp Duplicates & & & & & & & & & & & & & \\
\hline 39 & KS $11 \mathrm{D}$ & Soil & 1.1 & 55.3 & 9.6 & 36 & $<0.1$ & 51.9 & 20.6 & 1273 & 9.08 & 8.5 & 30 & 3.8 \\
\hline 40 & KS $11 \mathrm{D}$ & REP & 1.1 & 57 & 9.5 & 37 & $<0.1$ & 53.9 & 21.7 & 1241 & 9.4 & 8.6 & 20 & 3.9 \\
\hline 41 & KS8B & Soil & 0.8 & 91.4 & 14.6 & 281 & $<0.1$ & 77.4 & 102.3 & 7866 & 3.87 & 14.1 & 257.2 & 3.2 \\
\hline 42 & KS8B & REP & 0.8 & 90.8 & 14.6 & 282 & $<0.1$ & 77.4 & 100.3 & 8027 & 3.93 & 14.2 & 258.4 & 3.3 \\
\hline 42 & Reference Materials & & & & & & & & & & & & & \\
\hline 44 & STD DS10 & STD & 14 & 153.9 & 152.7 & 360 & 1.9 & 74 & 13.1 & 874 & 2.78 & 44.3 & 89.5 & 7.8 \\
\hline 45 & STD OXC109 & STD & 1.3 & 34.1 & 11.1 & 40 & $<0.1$ & 67.3 & 19.1 & 390 & 2.88 & 0.8 & 180.5 & 1.5 \\
\hline 46 & STD DS10 & STD & 14.4 & 148.6 & 152 & 326 & 2 & 71.6 & 12.7 & 840 & 2.58 & 43.3 & 88.8 & 7.9 \\
\hline 47 & STD OXC109 & STD & 1.5 & 34.7 & 11.8 & 39 & $<0.1$ & 69.5 & 19.1 & 385 & 2.71 & 0.7 & 189.1 & 1.6 \\
\hline 48 & BLK & BLK & $<0.1$ & $<0.1$ & $<0.1$ & $<1$ & $<0.1$ & $<0.1$ & $<0.1$ & $<1$ & $<0.01$ & $<0.5$ & $<0.5$ & $<0.1$ \\
\hline 50 & Prep Wash & & & & & & & & & & & & & \\
\hline 51 & G1 & Prep Blank & 0.1 & 3.1 & 3.6 & 45 & $<0.1$ & 3.5 & 4.1 & 517 & 1.79 & 0.5 & $<0.5$ & 5.8 \\
\hline \multirow[t]{5}{*}{52} & G1 & Prep Blank & 0.2 & 2.8 & 3.5 & 45 & $<0.1$ & 3.2 & 3.8 & 523 & 1.82 & $<0.5$ & 0.7 & 5.6 \\
\hline & & Analyte & $\mathrm{Sr}$ & $\mathrm{Cd}$ & Sb & $\mathbf{B i}$ & $\mathrm{V}$ & $\mathrm{Ca}$ & $\mathbf{P}$ & La & $\mathrm{Cr}$ & $\mathrm{Mg}$ & Ba & \\
\hline & & Unit & PPM & PPM & PPM & PPM & PPM & $\%$ & $\%$ & PPM & PPM & $\%$ & PPM & \\
\hline & & MDL & 1 & 0.1 & 0.1 & 0.1 & 2 & 0.01 & 0.001 & 1 & 1 & 0.01 & 1 & \\
\hline & Sample & Type & & & & & & & & & & & & \\
\hline 1 & KS1A & Soil & 3 & $<0.1$ & 0.3 & 0.1 & 42 & 0.02 & 0.014 & 6 & 64 & 0.02 & 54 & \\
\hline 2 & KS1B & Soil & 1 & $<0.1$ & 0.2 & 0.1 & 31 & $<0.01$ & 0.013 & 6 & 47 & 0.02 & 62 & \\
\hline 3 & KS1C & Soil & 4 & $<0.1$ & 0.4 & 0.2 & 46 & 0.02 & 0.014 & 7 & 67 & 0.02 & 74 & \\
\hline 4 & KS1D & Soil & $<1$ & $<0.1$ & 0.2 & 0.1 & 25 & $<0.01$ & 0.008 & 5 & 38 & 0.01 & 40 & \\
\hline 5 & KS2A & Soil & 3 & $<0.1$ & 0.3 & 0.1 & 39 & 0.01 & 0.011 & 6 & 52 & 0.03 & 113 & \\
\hline 6 & KS2B & Soil & 24 & 0.1 & 0.4 & 0.2 & 55 & 0.04 & 0.023 & 16 & 71 & 0.05 & 319 & \\
\hline 7 & KS2C & Soil & 52 & 0.2 & 0.5 & 0.4 & 56 & 0.02 & 0.015 & 15 & 65 & 0.03 & 245 & \\
\hline 8 & $\mathrm{KS} 2 \mathrm{C} 2$ & Soil & 52 & 0.1 & 0.5 & 0.4 & 53 & 0.03 & 0.015 & 14 & 60 & 0.03 & 239 & \\
\hline 9 & KS2D & Soil & 387 & 0.5 & 0.5 & 0.2 & 54 & 0.07 & 0.017 & 21 & 57 & 0.07 & 226 & \\
\hline 10 & KS3A1 & Soil & 4 & $<0.1$ & 0.2 & 0.1 & 85 & 0.02 & 0.02 & 10 & 107 & 0.02 & 67 & \\
\hline 11 & KS3A2 & Soil & 4 & $<0.1$ & 0.2 & 0.1 & 86 & 0.02 & 0.022 & 11 & 114 & 0.03 & 72 & \\
\hline 13 & KS3B & Soil & 22 & $<0.1$ & 0.3 & 0.1 & 88 & 0.02 & 0.021 & 11 & 100 & 0.03 & 113 & \\
\hline 14 & KS3C & Soil & 9 & $<0.1$ & 0.3 & 0.2 & 93 & 0.1 & 0.033 & 13 & 123 & 0.04 & 76 & \\
\hline
\end{tabular}


Table 2. Cont

\begin{tabular}{|c|c|c|c|c|c|c|c|c|c|c|c|c|c|}
\hline & & Analyte & $\mathrm{Sr}$ & $\mathrm{Cd}$ & $\mathrm{Sb}$ & Bi & V & $\mathrm{Ca}$ & $\mathbf{P}$ & La & $\mathrm{Cr}$ & Mg & Ba \\
\hline & & Unit & PPM & PPM & PPM & PPM & PPM & $\%$ & $\%$ & PPM & PPM & $\%$ & PPM \\
\hline & & MDL & 1 & 0.1 & 0.1 & 0.1 & 2 & 0.01 & 0.001 & 1 & 1 & 0.01 & 1 \\
\hline & Sample & Type & & & & & & & & & & & \\
\hline 15 & KS3D & Soil & 8 & $<0.1$ & 0.3 & 0.2 & 97 & 0.14 & 0.035 & 18 & 122 & 0.04 & 141 \\
\hline 16 & KS4A & Soil & 7 & 0.5 & 0.3 & 0.1 & 47 & 0.1 & 0.026 & 7 & 100 & 0.03 & 125 \\
\hline 17 & KS4B & Soil & 3 & $<0.1$ & 0.2 & $<0.1$ & 44 & 0.02 & 0.015 & 7 & 84 & 0.02 & 99 \\
\hline 18 & KS5A & Soil & 6 & $<0.1$ & 0.2 & 0.2 & 62 & 0.04 & 0.015 & 15 & 107 & 0.04 & 94 \\
\hline 19 & KS5B & Soil & 3 & $<0.1$ & 0.2 & 0.2 & 53 & 0.02 & 0.013 & 13 & 95 & 0.03 & 73 \\
\hline 20 & KS6A & Soil & 3 & $<0.1$ & 0.2 & $<0.1$ & 71 & 0.03 & 0.017 & 7 & 99 & 0.02 & 83 \\
\hline 21 & KS6B & Soil & 2 & $<0.1$ & 0.1 & $<0.1$ & 46 & 0.02 & 0.016 & 6 & 73 & 0.02 & 29 \\
\hline 22 & KS7A & Soil & 64 & 0.3 & 1.4 & 0.6 & 46 & 0.14 & 0.065 & 20 & 88 & 0.04 & 405 \\
\hline 23 & KS7B1 & Soil & 53 & 0.8 & 1 & 0.7 & 64 & 0.09 & 0.077 & 23 & 120 & 0.03 & 290 \\
\hline 24 & KS7B2 & Soil & 29 & 0.4 & 0.5 & 0.4 & 61 & 0.06 & 0.051 & 18 & 99 & 0.02 & 162 \\
\hline 25 & KS8A & Soil & 11 & 0.7 & 0.4 & 0.2 & 70 & 0.16 & 0.025 & 17 & 96 & 0.09 & 150 \\
\hline 26 & KS8B & Soil & 11 & 0.4 & 0.3 & 0.1 & 59 & 0.08 & 0.032 & 11 & 80 & 0.06 & 169 \\
\hline 27 & KS9A & Soil & 1 & $<0.1$ & 0.3 & 0.2 & 48 & $<0.01$ & 0.016 & 10 & 69 & 0.02 & 78 \\
\hline 28 & KS9B & Soil & 2 & $<0.1$ & 0.3 & 0.2 & 40 & 0.01 & 0.016 & 9 & 64 & 0.02 & 89 \\
\hline 29 & KS9C & Soil & 2 & $<0.1$ & 0.3 & 0.2 & 51 & 0.02 & 0.016 & 10 & 73 & 0.02 & 84 \\
\hline 30 & KS9D & Soil & 2 & $<0.1$ & 0.3 & 0.2 & 63 & 0.02 & 0.016 & 13 & 91 & 0.02 & 73 \\
\hline 31 & KS $10 \mathrm{~A}$ & Soil & 21 & 0.7 & 1.5 & 5.3 & 59 & 0.27 & 0.145 & 15 & 113 & 0.06 & 258 \\
\hline 32 & KS 11 Anthill sample & Soil & 4 & $<0.1$ & 0.4 & 0.2 & 70 & 0.04 & 0.046 & 12 & 176 & 0.03 & 32 \\
\hline 33 & KS $11 \mathrm{~A} 1$ & Soil & 2 & $<0.1$ & 0.5 & 0.2 & 42 & 0.01 & 0.019 & 6 & 79 & 0.02 & 15 \\
\hline 34 & KS 11 A2 & Soil & 2 & $<0.1$ & 0.7 & 0.2 & 40 & $<0.01$ & 0.018 & 6 & 76 & 0.02 & 14 \\
\hline 35 & KS 11 B & Soil & 2 & $<0.1$ & 0.2 & 0.1 & 24 & $<0.01$ & 0.013 & 5 & 49 & 0.01 & 9 \\
\hline 36 & KS $11 \mathrm{C}$ & Soil & 4 & $<0.1$ & 0.3 & 0.2 & 74 & 0.06 & 0.029 & 11 & 227 & 0.03 & 26 \\
\hline 37 & KS $11 \mathrm{D}$ & Soil & 3 & $<0.1$ & 0.5 & 0.2 & 82 & 0.01 & 0.042 & 15 & 233 & 0.03 & 38 \\
\hline 38 & *Pulp Duplicates & & & & & & & & & & & & \\
\hline 39 & KS $11 \mathrm{D}$ & Soil & 3 & $<0.1$ & 0.5 & 0.2 & 82 & 0.01 & 0.042 & 15 & 233 & 0.03 & 38 \\
\hline 40 & KS $11 \mathrm{D}$ & REP & 3 & $<0.1$ & 0.5 & 0.2 & 86 & 0.01 & 0.046 & 15 & 232 & 0.04 & 38 \\
\hline 41 & KS8B & Soil & 11 & 0.4 & 0.3 & 0.1 & 59 & 0.08 & 0.032 & 11 & 80 & 0.06 & 169 \\
\hline 42 & KS8B & REP & 11 & 0.4 & 0.4 & 0.1 & 60 & 0.1 & 0.034 & 12 & 87 & 0.06 & 174 \\
\hline 42 & Reference Materials & & & & & & & & & & & & \\
\hline 44 & STD DS10 & STD & 66 & 2.6 & 8.6 & 12.7 & 42 & 1.07 & 0.075 & 18 & 54 & 0.74 & 315 \\
\hline 45 & STD OXC109 & STD & 130 & $<0.1$ & $<0.1$ & $<0.1$ & 44 & 0.62 & 0.095 & 12 & 55 & 1.36 & 54 \\
\hline 46 & STD DS10 & STD & 68 & 2.7 & 9.4 & 12.6 & 40 & 1.02 & 0.075 & 18 & 52 & 0.75 & 348 \\
\hline 47 & STD OXC109 & STD & 138 & $<0.1$ & $<0.1$ & $<0.1$ & 46 & 0.68 & 0.104 & 12 & 56 & 1.43 & 57 \\
\hline 48 & BLK & BLK & $<1$ & $<0.1$ & $<0.1$ & $<0.1$ & $<2$ & $<0.01$ & $<0.001$ & $<1$ & $<1$ & $<0.01$ & $<1$ \\
\hline
\end{tabular}


Table 2. Cont

\begin{tabular}{|c|c|c|c|c|c|c|c|c|c|c|c|c|c|c|c|}
\hline & & Analyte & $\mathrm{Sr}$ & $\mathrm{Cd}$ & $\mathrm{Sb}$ & $\mathbf{B i}$ & $\mathrm{V}$ & $\mathrm{Ca}$ & $\mathbf{P}$ & La & $\mathrm{Cr}$ & $\mathrm{Mg}$ & Ba & & \\
\hline & & Unit & PPM & PPM & PPM & PPM & PPM & $\%$ & $\%$ & PPM & PPM & $\%$ & PPM & & \\
\hline & & MDL & 1 & 0.1 & 0.1 & 0.1 & 2 & 0.01 & 0.001 & 1 & 1 & 0.01 & 1 & & \\
\hline & Sample & Type & & & & & & & & & & & & & \\
\hline 50 & Prep Wash & & & & & & & & & & & & & & \\
\hline 51 & G1 & Prep Blank & 45 & $<0.1$ & $<0.1$ & $<0.1$ & 38 & 0.45 & 0.073 & 10 & 7 & 0.5 & 158 & & \\
\hline \multirow[t]{5}{*}{52} & G1 & Prep Blank & 47 & $<0.1$ & $<0.1$ & $<0.1$ & 37 & 0.45 & 0.066 & 10 & 7 & 0.5 & 155 & & \\
\hline & & Analyte & $\mathrm{Ti}$ & B & $\mathrm{Al}$ & $\mathrm{Na}$ & $\mathrm{K}$ & $\mathbf{W}$ & $\mathrm{Hg}$ & Sc & $\mathrm{Tl}$ & $\mathrm{S}$ & $\mathrm{Ga}$ & Se & Te \\
\hline & & Unit & $\%$ & PPM & $\%$ & $\%$ & $\%$ & PPM & PPM & PPM & PPM & $\%$ & PPM & PPM & PPM \\
\hline & & MDL & 0.001 & 1 & 0.01 & 0.001 & 0.01 & 0.1 & 0.01 & 0.1 & 0.1 & 0.05 & 1 & 0.5 & 0.2 \\
\hline & Sample & Type & & & & & & & & & & & & & \\
\hline 1 & KS1A & Soil & 0.017 & $<1$ & 0.86 & 0.002 & 0.03 & $<0.1$ & 0.03 & 4.7 & $<0.1$ & $<0.05$ & 5 & $<0.5$ & $<0.2$ \\
\hline 2 & KS1B & Soil & 0.014 & $<1$ & 0.82 & 0.002 & 0.02 & $<0.1$ & 0.03 & 3.7 & $<0.1$ & $<0.05$ & 4 & $<0.5$ & $<0.2$ \\
\hline 3 & KS1C & Soil & 0.017 & $<1$ & 0.99 & 0.004 & 0.02 & $<0.1$ & 0.04 & 4.7 & 0.1 & $<0.05$ & 5 & $<0.5$ & $<0.2$ \\
\hline 4 & KS1D & Soil & 0.01 & $<1$ & 0.57 & 0.003 & 0.02 & $<0.1$ & 0.02 & 2.6 & $<0.1$ & $<0.05$ & 3 & $<0.5$ & $<0.2$ \\
\hline 5 & KS2A & Soil & 0.018 & $<1$ & 1.05 & 0.003 & 0.03 & $<0.1$ & 0.03 & 4.6 & $<0.1$ & $<0.05$ & 5 & $<0.5$ & $<0.2$ \\
\hline 6 & KS2B & Soil & 0.029 & $<1$ & 1.81 & 0.003 & 0.06 & $<0.1$ & 0.04 & 6.3 & 0.1 & $<0.05$ & 8 & $<0.5$ & $<0.2$ \\
\hline 7 & KS2C & Soil & 0.04 & 1 & 1.43 & 0.004 & 0.06 & $<0.1$ & 0.07 & 8.6 & 0.1 & $<0.05$ & 8 & $<0.5$ & $<0.2$ \\
\hline 8 & $\mathrm{KS} 2 \mathrm{C} 2$ & Soil & 0.038 & 2 & 1.46 & 0.004 & 0.06 & 0.1 & 0.08 & 8.4 & 0.1 & $<0.05$ & 9 & $<0.5$ & $<0.2$ \\
\hline 9 & KS2D & Soil & 0.041 & $<1$ & 1.73 & 0.006 & 0.07 & 0.1 & 0.5 & 6.9 & 0.1 & $<0.05$ & 13 & $<0.5$ & $<0.2$ \\
\hline 10 & KS3A1 & Soil & 0.033 & $<1$ & 1.63 & 0.004 & 0.03 & $<0.1$ & 0.04 & 7.1 & $<0.1$ & $<0.05$ & 8 & $<0.5$ & $<0.2$ \\
\hline 11 & KS3A2 & Soil & 0.034 & $<1$ & 1.8 & 0.004 & 0.03 & $<0.1$ & 0.03 & 7.2 & $<0.1$ & $<0.05$ & 8 & $<0.5$ & $<0.2$ \\
\hline 13 & KS3B & Soil & 0.036 & $<1$ & 1.76 & 0.003 & 0.04 & $<0.1$ & 0.04 & 6.7 & $<0.1$ & $<0.05$ & 9 & $<0.5$ & $<0.2$ \\
\hline 14 & KS3C & Soil & 0.042 & 2 & 2.15 & 0.008 & 0.06 & $<0.1$ & 0.03 & 8.8 & $<0.1$ & $<0.05$ & 10 & $<0.5$ & $<0.2$ \\
\hline 15 & KS3D & Soil & 0.035 & 3 & 2.37 & 0.007 & 0.05 & $<0.1$ & 0.04 & 8.9 & $<0.1$ & $<0.05$ & 10 & $<0.5$ & $<0.2$ \\
\hline 16 & KS4A & Soil & 0.023 & 1 & 1.13 & 0.004 & 0.04 & $<0.1$ & 0.04 & 4.8 & 0.1 & $<0.05$ & 6 & $<0.5$ & $<0.2$ \\
\hline 17 & KS4B & Soil & 0.025 & $<1$ & 1.12 & 0.002 & 0.03 & $<0.1$ & 0.03 & 4.4 & $<0.1$ & $<0.05$ & 5 & $<0.5$ & $<0.2$ \\
\hline 18 & KS5A & Soil & 0.027 & $<1$ & 1.52 & 0.003 & 0.03 & $<0.1$ & 0.02 & 7 & 0.1 & $<0.05$ & 7 & $<0.5$ & $<0.2$ \\
\hline 19 & KS5B & Soil & 0.026 & $<1$ & 1.39 & 0.003 & 0.03 & $<0.1$ & 0.02 & 5.8 & $<0.1$ & $<0.05$ & 6 & $<0.5$ & $<0.2$ \\
\hline 20 & KS6A & Soil & 0.027 & $<1$ & 1.38 & 0.005 & 0.03 & $<0.1$ & 0.01 & 6.5 & 0.1 & $<0.05$ & 6 & $<0.5$ & $<0.2$ \\
\hline 21 & KS6B & Soil & 0.02 & $<1$ & 1.39 & 0.004 & 0.03 & $<0.1$ & 0.02 & 4.9 & $<0.1$ & $<0.05$ & 5 & $<0.5$ & $<0.2$ \\
\hline 22 & KS7A & Soil & 0.022 & 3 & 1.16 & 0.014 & 0.05 & 0.2 & 0.33 & 5 & 0.1 & 0.15 & 6 & 1 & $<0.2$ \\
\hline 23 & KS7B1 & Soil & 0.034 & 3 & 1.71 & 0.012 & 0.05 & 0.2 & 0.26 & 6.9 & 0.1 & 0.09 & 9 & 0.6 & $<0.2$ \\
\hline 24 & KS7B2 & Soil & 0.025 & 1 & 1.79 & 0.007 & 0.04 & 0.1 & 0.15 & 6.8 & $<0.1$ & 0.05 & 8 & $<0.5$ & $<0.2$ \\
\hline 25 & KS8A & Soil & 0.036 & 2 & 1.25 & 0.009 & 0.05 & $<0.1$ & 0.22 & 5.7 & 0.1 & $<0.05$ & 6 & $<0.5$ & $<0.2$ \\
\hline
\end{tabular}


Table 2. Cont.

\begin{tabular}{|c|c|c|c|c|c|c|c|c|c|c|c|c|c|c|c|}
\hline & & Analyte & $\mathrm{Ti}$ & B & Al & $\mathrm{Na}$ & $\mathbf{K}$ & W & $\mathrm{Hg}$ & Sc & $\mathrm{Tl}$ & $\mathrm{S}$ & Ga & Se & $\mathrm{Te}$ \\
\hline & & Unit & $\%$ & PPM & $\%$ & $\%$ & $\%$ & PPM & PPM & PPM & PPM & $\%$ & PPM & PPM & PPM \\
\hline & & MDL & 0.001 & 1 & 0.01 & 0.001 & 0.01 & 0.1 & 0.01 & 0.1 & 0.1 & 0.05 & 1 & 0.5 & 0.2 \\
\hline & Sample & Type & & & & & & & & & & & & & \\
\hline 26 & KS8B & Soil & 0.033 & 2 & 1.52 & 0.01 & 0.05 & $<0.1$ & 0.24 & 6.5 & 0.2 & 0.09 & 7 & $<0.5$ & $<0.2$ \\
\hline 27 & KS9A & Soil & 0.019 & $<1$ & 1.28 & 0.002 & 0.02 & $<0.1$ & 0.03 & 6 & 0.1 & $<0.05$ & 6 & $<0.5$ & $<0.2$ \\
\hline 28 & KS9B & Soil & 0.017 & $<1$ & 1.16 & 0.004 & 0.02 & $<0.1$ & 0.04 & 5.4 & 0.1 & $<0.05$ & 5 & $<0.5$ & $<0.2$ \\
\hline 29 & KS9C & Soil & 0.02 & $<1$ & 1.23 & 0.002 & 0.02 & $<0.1$ & 0.04 & 6.6 & 0.1 & $<0.05$ & 6 & $<0.5$ & $<0.2$ \\
\hline 30 & KS9D & Soil & 0.023 & $<1$ & 1.39 & 0.002 & 0.02 & $<0.1$ & 0.02 & 7 & $<0.1$ & $<0.05$ & 8 & 0.6 & $<0.2$ \\
\hline 31 & KS $10 \mathrm{~A}$ & Soil & 0.028 & 2 & 1.59 & 0.008 & 0.06 & 0.4 & 1.36 & 5.1 & 0.1 & $<0.05$ & 7 & 0.5 & $<0.2$ \\
\hline 32 & KS 11 Anthill sample & Soil & 0.025 & 1 & 1.57 & 0.006 & 0.04 & 0.1 & 0.06 & 8.8 & $<0.1$ & $<0.05$ & 8 & $<0.5$ & $<0.2$ \\
\hline 33 & KS 11 A1 & Soil & 0.014 & $<1$ & 0.93 & 0.007 & 0.03 & $<0.1$ & 0.04 & 3.1 & $<0.1$ & $<0.05$ & 4 & $<0.5$ & $<0.2$ \\
\hline 34 & KS 11 A2 & Soil & 0.013 & $<1$ & 0.93 & 0.006 & 0.03 & $<0.1$ & 0.03 & 3 & $<0.1$ & $<0.05$ & 4 & $<0.5$ & $<0.2$ \\
\hline 35 & KS 11 B & Soil & 0.008 & $<1$ & 0.65 & 0.007 & 0.03 & $<0.1$ & 0.03 & 1.8 & $<0.1$ & $<0.05$ & 2 & $<0.5$ & $<0.2$ \\
\hline 36 & KS $11 \mathrm{C}$ & Soil & 0.025 & $<1$ & 1.44 & 0.005 & 0.02 & $<0.1$ & 0.04 & 8.2 & $<0.1$ & $<0.05$ & 8 & $<0.5$ & $<0.2$ \\
\hline 37 & KS $11 \mathrm{D}$ & Soil & 0.029 & $<1$ & 2 & 0.005 & 0.04 & 0.2 & 0.05 & 11.5 & 0.1 & $<0.05$ & 10 & $<0.5$ & $<0.2$ \\
\hline 38 & *Pulp Duplicates & & & & & & & & & & & & & & \\
\hline 39 & KS $11 \mathrm{D}$ & Soil & 0.029 & $<1$ & 2 & 0.005 & 0.04 & 0.2 & 0.05 & 11.5 & 0.1 & $<0.05$ & 10 & $<0.5$ & $<0.2$ \\
\hline 40 & KS $11 \mathrm{D}$ & REP & 0.029 & $<1$ & 1.93 & 0.005 & 0.04 & 0.3 & 0.06 & 11.1 & 0.1 & $<0.05$ & 11 & $<0.5$ & $<0.2$ \\
\hline 41 & KS8B & Soil & 0.033 & 2 & 1.52 & 0.01 & 0.05 & $<0.1$ & 0.24 & 6.5 & 0.2 & 0.09 & 7 & $<0.5$ & $<0.2$ \\
\hline 42 & KS8B & REP & 0.037 & 3 & 1.67 & 0.01 & 0.06 & $<0.1$ & 0.26 & 6.8 & 0.2 & 0.1 & 7 & $<0.5$ & $<0.2$ \\
\hline 42 & Reference Materials & & & & & & & & & & & & & & \\
\hline 44 & STD DS10 & STD & 0.073 & 6 & 0.99 & 0.062 & 0.32 & 3.2 & 0.29 & 2.8 & 5.2 & 0.26 & 4 & 2.2 & 4.9 \\
\hline 45 & STD OXC109 & STD & 0.332 & 1 & 1.42 & 0.611 & 0.37 & 0.2 & $<0.01$ & 1 & $<0.1$ & $<0.05$ & 5 & $<0.5$ & $<0.2$ \\
\hline 46 & STD DS10 & STD & 0.077 & 7 & 0.98 & 0.065 & 0.31 & 3.5 & 0.28 & 2.7 & 4.8 & 0.26 & 5 & 2.2 & 5.3 \\
\hline 47 & STD OXC109 & STD & 0.387 & 2 & 1.46 & 0.654 & 0.4 & 0.2 & $<0.01$ & 1 & $<0.1$ & $<0.05$ & 5 & $<0.5$ & $<0.2$ \\
\hline 48 & BLK & BLK & $<0.001$ & $<1$ & $<0.01$ & $<0.001$ & $<0.01$ & $<0.1$ & $<0.01$ & 0.2 & $<0.1$ & $<0.05$ & $<1$ & $<0.5$ & $<0.2$ \\
\hline 50 & Prep Wash & & & & & & & & & & & & & & \\
\hline 51 & G1 & Prep Blank & 0.105 & $<1$ & 0.86 & 0.066 & 0.46 & $<0.1$ & $<0.01$ & 2.3 & 0.2 & $<0.05$ & 4 & $<0.5$ & $<0.2$ \\
\hline 52 & G1 & Prep Blank & 0.099 & $<1$ & 0.89 & 0.064 & 0.45 & $<0.1$ & $<0.01$ & 2.1 & 0.3 & $<0.05$ & 4 & $<0.5$ & $<0.2$ \\
\hline
\end{tabular}


Table 3. Full table of selected heavy metal concentrations $\left(\mathrm{mg} \cdot \mathrm{kg}^{-1}\right.$ ) in the Krugersdorp Game Reserve, Rand Uranium Mine, Mintails Mogale Gold and surrounding smallholdings.

\begin{tabular}{|c|c|c|c|c|c|c|c|c|c|c|c|c|}
\hline \multicolumn{2}{|c|}{ Heavy Metal } & \multicolumn{7}{|c|}{ Krugersdorp Game Reserve } & \multirow{2}{*}{$\begin{array}{c}\begin{array}{c}\text { Rand } \\
\text { Uranium } \\
\text { Mine }\end{array} \\
\text { KS6 } \\
(n=2)\end{array}$} & \multirow{2}{*}{$\begin{array}{c}\begin{array}{c}\text { Mintails } \\
\text { Mogale } \\
\text { Gold }\end{array} \\
\text { KS7 } \\
(n=3)\end{array}$} & \multicolumn{2}{|c|}{ Smallholdings } \\
\hline & & $\begin{array}{c}\text { KS1 } \\
(n=4)\end{array}$ & $\begin{array}{c}\text { KS2 } \\
(n=5)\end{array}$ & $\begin{array}{c}\text { KS3 } \\
(n=5)\end{array}$ & $\begin{array}{c}\text { KS8 } \\
(n=2)\end{array}$ & $\begin{array}{c}\text { KS9 } \\
(n=4)\end{array}$ & $\begin{array}{l}\text { KS10 } \\
(n=1)\end{array}$ & $\begin{array}{c}\text { KS11 } \\
(n=6)\end{array}$ & & & $\begin{array}{c}\text { KS4 } \\
(n=2)\end{array}$ & $\begin{array}{c}\text { KS5 } \\
(n=2)\end{array}$ \\
\hline \multirow{4}{*}{$\begin{array}{c}\mathrm{Cr} \\
(90.00) *\end{array}$} & Mean & 56.25 & 61.00 & 113.20 & 88.00 & 74.25 & 113.00 & 140.00 & 86.00 & 102.33 & 92.00 & 101.00 \\
\hline & SD & 17.02 & 7.31 & 9.83 & 11.31 & 11.76 & N/A & 81.99 & 18.39 & 16.26 & 11.31 & 8.49 \\
\hline & Min & 38.00 & 52.00 & 100.00 & 80.00 & 64.00 & N/A & 49.00 & 73.00 & 88.00 & 84.00 & 95.00 \\
\hline & Max & 67.00 & 71.00 & 123.00 & 96.00 & 91.00 & N/A & 233.00 & 99.00 & 120.00 & 100.00 & 107.00 \\
\hline \multirow{4}{*}{$\begin{array}{c}\text { Mn } \\
(850.00)\end{array}$} & Mean & 2564.00 & 9011.20 & 4635.00 & 8578.00 & 1980.75 & 8990.00 & 502.17 & 631.50 & 3938.00 & 5435.00 & 1320.00 \\
\hline & SD & 553.94 & 2211.02 & 2596.11 & 1006.92 & 667.08 & N/A & 425.64 & 255.27 & 2801.97 & 3124.00 & 336.58 \\
\hline & Min & 1801.00 & 5056.00 & 2956.00 & 7866.00 & 1122.00 & N/A & 115.00 & 451.00 & 1717.00 & 3226.00 & 1082.00 \\
\hline & Max & 3128.00 & $10,000.00$ & 9233.00 & 9290.00 & 2628.00 & N/A & 1273.00 & 812.00 & 7086.00 & 7644.00 & 1558.00 \\
\hline \multirow{4}{*}{$\begin{array}{c}\text { Co } \\
(19.00) *\end{array}$} & Mean & 10.78 & 14.62 & 11.76 & 75.50 & 11.58 & 39.40 & 9.57 & 31.10 & 41.20 & 51.70 & 8.90 \\
\hline & SD & 1.01 & 2.69 & 1.36 & 37.90 & 2.29 & N/A & 6.81 & 21.07 & 12.38 & 58.41 & 3.54 \\
\hline & Min & 9.30 & 10.50 & 10.80 & 48.70 & 9.00 & N/A & 2.80 & 16.20 & 26.90 & 10.40 & 6.40 \\
\hline & Max & 11.60 & 18.00 & 14.10 & 102.30 & 13.70 & $\mathrm{~N} / \mathrm{A}$ & 20.60 & 46.00 & 48.40 & 93.00 & 11.40 \\
\hline \multirow{4}{*}{$\begin{array}{c}\mathrm{Ni} \\
(68.00)\end{array}$} & Mean & 24.05 & 153.74 & 55.08 & 93.80 & 29.75 & 117.60 & 30.52 & 45.60 & 81.33 & 148.15 & 20.65 \\
\hline & $\mathrm{SD}$ & 5.58 & 164.56 & 5.47 & 23.19 & 1.73 & N/A & 15.16 & 22.49 & 19.58 & 176.71 & 1.48 \\
\hline & Min & 16.40 & 38.50 & 49.30 & 77.40 & 27.50 & $\mathrm{~N} / \mathrm{A}$ & 13.90 & 29.70 & 59.60 & 23.20 & 19.60 \\
\hline & Max & 29.50 & 444.80 & 61.40 & 110.20 & 31.60 & N/A & 51.90 & 61.50 & 97.60 & 273.10 & 21.70 \\
\hline \multirow{4}{*}{$\begin{array}{c}\mathrm{Cu} \\
(45.00)\end{array}$} & Mean & 13.43 & 28.82 & 43.44 & 85.70 & 20.05 & 93.20 & 31.17 & 16.10 & 99.73 & 21.65 & 18.10 \\
\hline & SD & 2.94 & 9.21 & 3.34 & 8.06 & 2.81 & N/A & 17.79 & 3.39 & 33.76 & 11.53 & 2.83 \\
\hline & Min & 10.10 & 16.00 & 40.60 & 80.00 & 17.60 & $\mathrm{~N} / \mathrm{A}$ & 11.10 & 13.70 & 61.00 & 13.50 & 16.10 \\
\hline & Max & 16.00 & 42.00 & 49.00 & 91.40 & 24.00 & N/A & 55.30 & 18.50 & 115.30 & 29.80 & 20.10 \\
\hline \multirow{4}{*}{$\begin{array}{c}\mathrm{Zn} \\
(95.00)\end{array}$} & Mean & 17.75 & 91.60 & 38.00 & 508.50 & 18.25 & 446.00 & 22.67 & 23.50 & 657.33 & 107.00 & 12.50 \\
\hline & SD & 3.77 & 73.38 & 14.71 & 321.73 & 2.22 & N/A & 10.58 & 12.02 & 488.82 & 134.35 & 0.71 \\
\hline & Min & 15.00 & 25.00 & 25.00 & 281.00 & 16.00 & N/A & 10.00 & 15.00 & 153.00 & 12.00 & 12.00 \\
\hline & Max & 23.00 & 216.00 & 54.00 & 736.00 & 21.00 & N/A & 36.00 & 32.00 & 1129.00 & 202.00 & 13.00 \\
\hline
\end{tabular}


Table 3. Cont.

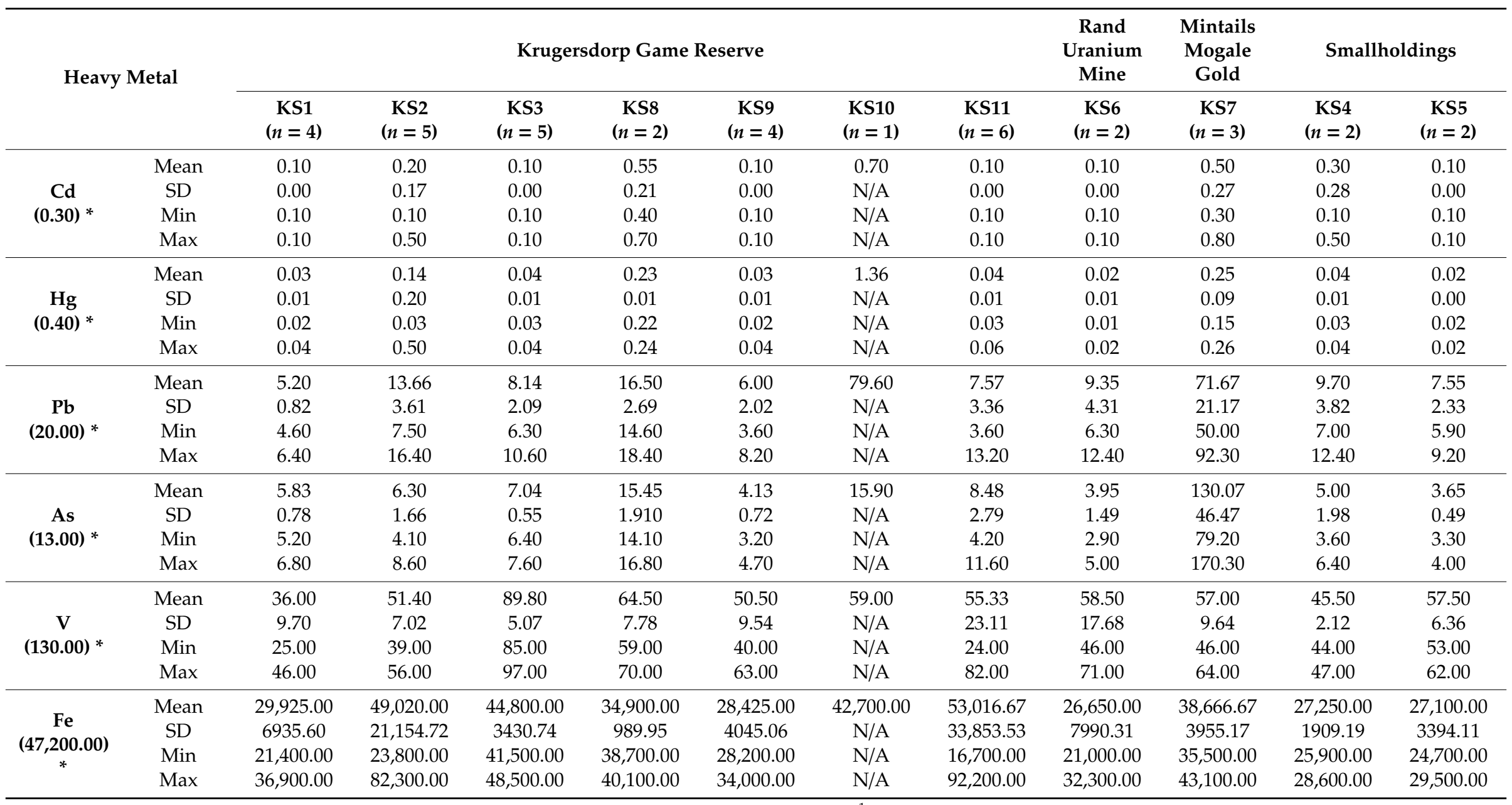

* The mean concentrations were compared to reference values $\left(\mathrm{mg} \cdot \mathrm{kg}^{-1}\right)$ for background concentrations after [26]. 
The sampling sites in the KGR (Table 3) showed a distinct migration pattern of heavy metal concentrations. Sampling sites KS8 and KS10 displayed higher mean concentrations than the other sampling sites in the KGR. This is interesting since KS8 and KS10 were located in close proximity to the TLS (Figure 1). The higher mean concentrations are to be expected since AMD first decanted into this waterway in September 2002 [28]. The sampling site KS1 in the KGR had predominantly the lowest mean heavy metal concentrations (Table 4). Again, this is to be expected, since this sampling site was the furthest from the point source of discharge (Figure 1).

\subsection{Distribution of Soil Geochemical Data}

Mean concentration ranges of Aqua Regia extractable $\mathrm{As}, \mathrm{Co}, \mathrm{Cu}, \mathrm{Hg}$ and $\mathrm{Pb}$ fraction (pseudo-total fraction) of soil samples collected for this study were compared with the range of maximum allowable concentrations (MAC) for agricultural soils worldwide, as given in [29]. Quite unexpectedly, mean PHE concentrations in soils of the KGR and SH are well within the range of MAC for agricultural soils worldwide. Table 4 gives the statistical parameters of surface soil PHE data, including standard deviations and location of mean contents within the range of MAC given for agricultural soils worldwide [29]. High standard deviations signify wider variations in PHE distribution from the point source of discharge of AMD to the adjacent areas. Of the four study segments (KGR, SH, MMG and $\mathrm{RU})$, only MMG gives a mean value for As (130.07 ppm) falling above the MAC range for agricultural soils worldwide (15-20 ppm). Mean As values for the other three segments fall well within the MAC range (Table 4). Similarly, Co, KGR and SH gave mean values for surface soils (102.30 ppm) and (93.00 ppm) respectively, that is above the upper threshold of the MAC range for agricultural soils (20-50 ppm) given in [29]. The other two study segments gave mean values well within this range.

The mean $\mathrm{Cu}$ content in surface soils of all four segments studied falls well within the MAC range for agricultural soils (60-150 ppm) given in [30]. The highest $\mathrm{Cu}$ values were again found in samples from the MMG segment, but with a mean value (99.73) far removed from the upper threshold (150 ppm) of the MAC range. The mean Cu concentrations decrease in the order: MMG (99.73 ppm) > KGR (35.07 ppm) > SH (19.88 ppm) > RU (16.10 ppm) (Table 4).

The mean $\mathrm{Hg}$ content in surface soils of all four segments studied falls well within the MAC range given for agricultural soils (0.5-5 ppm) in reference [29]. The highest $\mathrm{Hg}$ values were again found in samples from the MMG segment.

The mean $\mathrm{Pb}$ content in surface soils of all four segments studied falls well within the MAC range for $\mathrm{Pb}$ in agricultural soils (20-300 ppm) given in reference [29]. The highest $\mathrm{Pb}$ values were again found in samples from the MMG segment.

\subsection{Sampling Sites}

KS8 and KS10, in close proximity to the Tweelopiespruit (TLS) (Figure 1), displayed higher mean concentrations of PHEs in surface soil compared to the other sampling sites in the KGR. This is unsurprising, given that the TLS is the waterway where AMD first decanted in September 2002 [14]. The sampling site KS1 in the KGR gave the lowest PHE concentrations. Again, this is to be expected, since this sampling site was the furthest from the point source of AMD discharge (Figure 2). The relatively low concentrations of $\mathrm{Cu}, \mathrm{Hg}$ and $\mathrm{Pb}$ in surface soils of the KGR, explained both on the basis of improved quality of influent mine water and carbonate-water interactions in the soil, is taken to indicate that the potential supply of these elements to herbage and crops would be limited. 
Table 4. As, $\mathrm{Co}, \mathrm{Cu}, \mathrm{Hg}$ and $\mathrm{Pb}(\mathrm{ppm})$ in agricultural soils and in living matter, compared with mean values in soils of the study localities. ${ }^{\mathbf{a}}$ [29]; ${ }^{\mathbf{b}}$ [30].

\begin{tabular}{|c|c|c|c|c|c|c|c|c|}
\hline \multicolumn{9}{|c|}{ Study Sites } \\
\hline & PHE & $\begin{array}{l}\text { Krugersdorp } \\
\text { Game Reserve }\end{array}$ & Smallholdings & $\begin{array}{l}\text { Rand Uranium } \\
\text { Mine }\end{array}$ & $\begin{array}{l}\text { Mintails } \\
\text { Mogale Gold }\end{array}$ & $\begin{array}{l}\text { Range of MAC } \\
\text { in Agricultural } \\
\text { Soils }^{\text {a }}\end{array}$ & $\begin{array}{l}\text { Average } \\
\text { Composition of } \\
\text { Living Matter } b\end{array}$ & Comments \\
\hline \multirow{5}{*}{ As } & Mean & 7.56 & 4.33 & 3.95 & 130.07 & \multirow{5}{*}{$15-20$} & \multirow{5}{*}{0.31} & \multirow{5}{*}{$\begin{array}{l}\text { Toxic; readily absorbed by plants } \\
\text { from groundwater and soil }\end{array}$} \\
\hline & SD & 3.54 & 1.41 & 1.49 & 46.47 & & & \\
\hline & Min & 3.20 & 3.30 & 2.90 & 79.20 & & & \\
\hline & Max & 16.80 & 6.40 & 5.00 & 170.30 & & & \\
\hline & $\mathrm{N}$ & 23 & 4 & 2 & 2 & & & \\
\hline \multirow{5}{*}{ Co } & Mean & 17.37 & 30.30 & 31.10 & 41.20 & \multirow{5}{*}{$20-50$} & \multirow{5}{*}{0.2} & \multirow{5}{*}{$\begin{array}{l}\text { Microorganisms (Rhizobium sp.); } \\
\text { essential to animals }\end{array}$} \\
\hline & SD & 19.45 & 41.86 & 21.07 & 12.38 & & & \\
\hline & Min & 2.80 & 6.40 & 16.20 & 26.90 & & & \\
\hline & $\operatorname{Max}$ & 102.30 & 93.00 & 46.00 & 48.40 & & & \\
\hline & $\mathrm{N}$ & 23 & 4 & 2 & 2 & & & \\
\hline \multirow{5}{*}{$\mathrm{Cu}$} & Mean & 35.07 & 19.88 & 16.10 & 99.73 & \multirow{5}{*}{ 60-150 } & \multirow{5}{*}{2} & \multirow{5}{*}{$\begin{array}{l}\text { Vital to health of all living organisms. } \\
\text { Necessary for haemoglobin and } \\
\text { melanin formation; component of } \\
\text { several blood proteins and enzyme } \\
\text { systems }\end{array}$} \\
\hline & SD & 23.21 & 7.15 & 3.39 & 33.76 & & & \\
\hline & Min & 10.10 & 13.50 & 13.70 & 61.00 & & & \\
\hline & Max & 93.20 & 29.80 & 18.50 & 115.30 & & & \\
\hline & $\mathrm{N}$ & 23 & 4 & 2 & 2 & & & \\
\hline \multirow{5}{*}{$\mathrm{Hg}$} & Mean & 0.12 & 0.03 & 0.02 & 0.25 & \multirow{5}{*}{$0.5-5$} & \multirow{5}{*}{0.00} & \multirow{5}{*}{$\begin{array}{l}\text { Toxic. Fish can contain unusual } \\
\text { amounts }\end{array}$} \\
\hline & SD & 0.27 & 0.01 & 0.01 & 0.09 & & & \\
\hline & Min & 0.02 & 0.02 & 0.01 & 0.15 & & & \\
\hline & $\operatorname{Max}$ & 1.36 & 0.04 & 0.02 & 0.26 & & & \\
\hline & $\mathrm{N}$ & 23 & 4 & 2 & 2 & & & \\
\hline \multirow{5}{*}{$\mathrm{Pb}$} & Mean & 11.55 & 8.63 & 9.35 & 71.67 & \multirow{5}{*}{$20-300$} & \multirow{5}{*}{0.51} & \multirow{5}{*}{$\begin{array}{l}\text { Present in plants and animals; easily } \\
\text { absorbed and accumulates in } \\
\text { different plant parts }\end{array}$} \\
\hline & SD & 14.26 & 2.87 & 4.31 & 21.17 & & & \\
\hline & Min & 3.60 & 5.90 & 6.30 & 50.00 & & & \\
\hline & Max & 79.60 & 12.40 & 12.40 & 92.30 & & & \\
\hline & $\mathrm{N}$ & 23 & 4 & 2 & 2 & & & \\
\hline
\end{tabular}




\section{Conclusions}

Data from the hydrochemical literature as well as from current research on soil geochemistry indicate that large amounts of mine pollutants produced predominantly in the form of AMD have infiltrated soils and other environmental compartments of the KGR through ground- and surface waters, over several decades of gold mining. Analysis of the soils showed high levels of contamination for As and Co in ppm. The mean maximum of As ranged from (5.00-170.30) with the highest level found in the Krugersdorp site. The mean maximum of Co ranged from (46.00-102.30) with the highest level found in MMG. All of these values were well above the MAC recommended values, i.e., As (15-20) and $\mathrm{Co}$ (20-50). The mean maximum values for $\mathrm{Pb}$ (12.40-92.30), $\mathrm{Cu}$ (18.50-115.30), and $\mathrm{Hg}$ (12.40-92.30) content in surface soils of all four segments studied falls well within the MAC range for agricultural soils, i.e., $\mathrm{Cu}$ (60-150), $\mathrm{Hg}$ (0.5-5), and $\mathrm{Pb}$ (20-300). The major mediating influences on the behaviour of $\mathrm{As}, \mathrm{Co}, \mathrm{Cu}, \mathrm{Hg}$ and $\mathrm{Pb}$, as they enter the KGR largely in the form of acid mine drainage (AMD), are the geological substrate (mostly in carbonate form).

These pollutants can cause modifications to natural element cycling in the KGR-and adjacent soil environments. The fluxes of residual $\mathrm{As}, \mathrm{Co}, \mathrm{Cu}, \mathrm{Hg}$ and $\mathrm{Pb}$ in soils from influent AMD that is available for plant uptake in KGR is considered to be determined largely by the nature of the underlying substrate (predominantly carbonate) and the level of treatment of decant from adjacent mining localities during any given season. The high As and Co contents found in most of the soils in our study areas provide a major motivation for proposing further investigation of the waters infiltrated by AMD contamination and comparing it to the soil values obtained to give a more comprehensive idea of the amount of PHE accumulation. Well-designed, quantitative research along these lines will enable the rehabilitation of metal-contaminated areas with appropriate indigenous species, allow identification of metal toxicities when they occur and allow for the effective regulation of metal emissions. This information is considered vital in the formulation of intervention measures such as mitigation, Acid mine drainage (AMD) neutralization, rehabilitation and soil amendment. Results from these kinds of studies would enable more informed decisions to be taken on site investigations, clean-up actions, and remedy selection. In accordance with the observations of DEAT [31], more research is required on the field impact of AMD on potentially receiving dolomitic (karst) environments and the re-activation of springs dried-up due to dewatering. Strategies should be evolved to utilize the storage potential of defunct/closed underground mine voids so as to optimally manage the generation of AMD and control its potential impact on the receiving surface and groundwater environments.

Author Contributions: Funding acquisition and conceptualization, M.S. and T.C.D.; data curation and writing-original draft preparation and review and editing, M.S., M.A.J. and M.R.L.; formal analysis and preparation for sending to Canada, D.S.N., T.C.D. and M.S.; Maps, E.C. and M.D. All authors have read and agreed to the published version of the manuscript.

Funding: This research was funded by the Swedish International Development Agency (SIDA) Extra-Budgetary Funding for 2013 which was an additional grant awarded for the implementation of a proposed extension phase of IGCP/SIDA/UNESCO/MUT Project 606. The work was also supported by the Research Directorate and the Faculty of Natural Sciences, both of Mangosuthu University of Technology, South Africa.

Acknowledgments: We would like to thank the Management and Staff of both Mintails Mogale Gold and the Krugersdorp Game Reserve, for allowing access to their various properties, permitting the conduct of geochemical sampling and for holding group discussions at their premises. We acknowledge the professionalism shown by Bureau Veritas in Johannesburg, South Africa and ACME Laboratories in Vancouver, Canada in performing analytical work of the highest quality. In addition, we thank K.K. Naidoo for his valuable input and suggestions towards this study.

Conflicts of Interest: The authors declare no conflict of interest. 


\section{References}

1. Nadasan, D.S.; Davies, T.C.; Shapi, M.; Chirenje, E. The distribution of some potentially harmful elements (PHEs) in the Krugersdorp Game Reserve, Gauteng, South Africa: Implications for wildlife health. In Proceedings of the IGCP/SIDA Projects 594 and 606, Closing Workshop, Prague, Czech Republic, 26-28 May 2014.

2. Singo, N.K. An Assessment of Heavy Metal Pollution Near an Old Copper Mine Dump in Musina, South Africa. Ph.D. Thesis, University of South Africa, Pretoria, South Africa, 2013.

3. Pereira, S.I.A.; Lima, A.I.G.; Figueira, E.M.A.P. Heavy metal toxicity in Rhizobium leguminosarum biovar viciae isolated from soils subjected to different sources of heavy metal contamination: Effect on protein expression. Appl. Soil Ecol. 2006, 33, 286-293. [CrossRef]

4. Khan, M.S.; Zaidi, A.; Oves, M.; Wani, P.A. Heavy metal toxicity to legumes. In Heavy Metal Pollution; Samuel, E.B., William, C.W., Eds.; Nova Science: Hauppauge, NY, USA, 2008; pp. 197-225.

5. Zang, H.; Luo, Y.; Makino, T.; Wu, L.; Nanzyo, M. The heavy metal partition in size fractions of the fine particles in agricultural soils contaminated by waste water and smelter dust. J. Hazard. Mater. 2013, 248, 303-312. [CrossRef] [PubMed]

6. Saxena, P.K.; KrishnaRaj, S.; Perras, M.R.; Vettakkorumakankav, N.N. Phytoremediation of heavy metal contaminated and polluted soils. In Heavy Metal Stress in Plants; Springer: Berlin, Germany, 1999; pp. $305-329$.

7. Battaglia, A.; Ghidini, S.; Campanini, G.; Spaggiari, R. Heavy metal contamination in little owl (Athene noctua) and common buzzard (Buteo buteo) from northern Italy. Ecotoxicol Environ. Saf. 2005, 60, 61-66. [CrossRef] [PubMed]

8. Madejón, E.; Pérez de Mora, A.; Felipe, E.; Burgos, P.; Cabrera, F. Soil amendments reduce trace element solubility in a contaminated soil and allow regrowth of natural vegetation. Environ. Pollut. 2006, 139, 40-52. [CrossRef] [PubMed]

9. Adriano, D.C. Trace Elements in Terrestrial Environments: Biogeochemistry, Bioavailability and Risks of Metals, 2nd ed.; Springer: New York, NY, USA, 2001; Volume 867, pp. 219-796. [CrossRef]

10. Tangahu, B.V.; Abdullah, S.; Basri, H.; Idris, M.; Anuar, N.; Mukhlisin, M. A review on heavy metals (As, Pb, and $\mathrm{Hg}$ ) uptake by plants through phytoremediation. Int. J. Chem. Eng. 2011, 2011, 1-31. [CrossRef]

11. Akcil, A.; Koldas, S. Acid Mine Drainage (AMD): Causes, treatment and case studies. J. Clean. Prod. 2006, 14, 1139-1145. [CrossRef]

12. Winde, F. Uranium pollution of the Wonderfonteinspruit, 1997-2008 Part 1: Uranium toxicity, regional background and mining-related sources of uranium pollution. SA J. Radiol. 2010, 36, 13.

13. Paterson, D.G. Soils and Agricultural Potential Along the Proposed Westgate-Tarlton-Kromdraai Power Line, Gauteng Province, South Africa. Report for Arcus Gibb; Report No. GW/A/2008/10; Institute for Soil Climate and Water of the Agricultural Research Council. 2008. Available online: http://projects.gibb.co.za/Portals/3/projects/200902\%20Tarlton\%20Kromdraai\%20power/Environmental\% 20Impact $\% 20$ Phase/Appendix\%20H\%20\%20Agricultural\%20Potential\%20and\%20Soils\%20Study.pdf (accessed on 9 March 2016).

14. Darnley, A.G.; Björklund, A.; Bølviken, B.; Gustavsson, N.; Koval, P.V.; Plant, J.A.; Steenfelt, A.; Tauchid, M.; Xie, X. A Global Geochemical Database for Environmental and Resource Management: Recommendations for International Geochemical Mapping; Final Report of IGCP Project 259; UNESCO Publishing: Paris, France, 1995.

15. Leduc, C.; Itard, Y. Low sampling density exploration geochemistry for gold in arid and tropical climates: Comparison between conventional geochemistry and BLEG. Geochem. Explor. Environ. Anal. 2003, 3, 121-131. [CrossRef]

16. Ottosen, L.M.; Jensen, P.E. Electro-remediation of heavy-metal contaminated soil. In Soil and Sediment Remediation; IWA publishing: London, UK, 2005; pp. 264-288.

17. Jung, M.C.; Thornton, I. Heavy metal contamination of soils and plants in the vicinity of a lead-zinc mine, Korea. Appl. Geochem. 1996, 11, 53-59. [CrossRef]

18. Wang, H.-H.; Li, L.-Q.; Wu, X.M.; Pan, G.-X. Distribution of $\mathrm{Cu}$ and $\mathrm{Pb}$ in particle size fractions of urban soils from different city zones of Nanjing, China. J. Environ. Sci. 2006, 18, 482-487.

19. ECOMED Eastern Europe SRL. Contaminated Soil Remediation. Available online: http://www.ecomed-intl. com/contaminated-soil-remediation.html (accessed on 5 March 2016). 
20. Li, Q.; Ji, H.; Qin, F.; Tang, L.; Guo, X.; Feng, J. Sources and the distribution of heavy metals in the particle size of soil polluted by gold mining upstream of Miyun Reservoir, Beijing: Implications for assessing the potential risks. Environ. Monit. Assess. 2014, 186, 6605-6626. Available online: http: //dx.doi.org/10.1007/s10661-014-3877-4 (accessed on 5 March 2016). [CrossRef] [PubMed]

21. Shuman, L.M. Chemical forms of micronutrients in soil. In Micronutrients in Agriculture; Mortvedt, J.J., Cox, F.R., Shuman, L.M., Welch, R.M., Eds.; Soil Science Society of America: Madison, WI, USA, 1991.

22. Farmaki, S.; Karakasi, O.; Moutsatsu, A. $\mathrm{Pb}^{2+}$ and $\mathrm{Ni}^{2+}$ Adsorption on Limestone and Dolomite Tailings. Inżynieria Mineralna - Lipiec-Grudzień, Journal of the Polish Mineral Engineering Society, July-December, 2014. Available online: http://www.potopk.com.pl/Full_text/2014_full/2014_2_35.pdf (accessed on 24 February 2016).

23. Krishnamurti, G.S.R. Chemical methods for assessing contaminant bioavailability in soils. In Chemical Bioavailability in Terrestrial Environments, 1; Naidu, R., Ed.; Elsevier: Oxford, UK, 2008; pp. 495-520. ISBN 978-0-444-52169.

24. Du Toit, S. Practical applications-effects of mine water drainage on the Krugersdorp Game Reserve. In Proceedings of the Conference on Mine Water Decant, Randfontein, South Africa, 14-15 October 2006; Mine Water Division, Water Institute of South Africa: Midrand, South Africa, 2006.

25. Johnson, C.C.; Cave, M.R.; Palumbo-Roe, B.; Nathanail, C.P.; Lark, R.M. Methodology for the determination of normal background concentrations of contaminants in English soil. Sci. Total Environ. 2013, 454, 604-618.

26. Wedepohl, K.H. The composition of the continental crust. Geochim. Cosmochim. Acta 1995, 59, 1217-1232. [CrossRef]

27. Stream Destroyed by Acid Mine Water. Available online: http://earthlife.org.za/stream-destroyed-by-acidmine-water/ (accessed on 12 June 2016).

28. Ure, A.M.; Davidson, C.M. (Eds.) Chemical speciation in soils and related materials by selective chemical extraction. In Chemical Speciation in the Environment, 2nd ed.; Blackwell Science Ltd.: Oxford, UK, 2002.

29. Kabata-Pendias, A. Trace Elements in Soils and Plants; CRC Press: Boca Raton, FL, USA, 2000.

30. Perel'man, A.I. Geochemistry of Epigenesis; Springer: Berlin, Germany, 1967; p. 266.

31. DEAT (Department of Environmental Affairs and Tourism of South Africa). Mine Water Pollution-Acid mine Decant, Effluent and Treatment: A Consideration of Key Emerging Issues That May Impact the State of the Environment. Emerging Issues Paper: Mine Water Pollution 2008. Available online: http: //www.hsph.harvard.edu/mining/files/South_Africa.pdf (accessed on 1 March 2016). 